Journal for ImmunoTherapy of Cancer

\title{
Domain binding and isotype dictate the activity of anti-human OX40 antibodies
}

\author{
Jordana Griffiths, ${ }^{1}$ Khiyam Hussain, ${ }^{1}$ Hannah L Smith, ${ }^{1}$ Theodore Sanders, ${ }^{1}$ \\ Kerry L Cox, ${ }^{1}$ Monika Semmrich, ${ }^{2}$ Linda Mårtensson, ${ }^{2}$ Jinny Kim, ${ }^{1}$ \\ Tatyana Inzhelevskaya, ${ }^{1}$ Chris A Penfold, ${ }^{1}$ Alison L Tutt, ${ }^{1} \mathrm{C}$ lan Mockridge, ${ }^{1}$ \\ HT Claude Chan, ${ }^{1}$ Vikki English, ${ }^{1}$ Ruth F French, ${ }^{1}$ Ingrid Teige, ${ }^{2}$ \\ Aymen Al-Shamkhani, ${ }^{1}$ Martin J Glennie, ${ }^{1}$ Bjorn L Frendeus, ${ }^{2}$ \\ Jane E Willoughby (D) , ${ }^{1}$ Mark S Cragg (D) ${ }^{1}$
}

To cite: Griffiths J, Hussain K, Smith $\mathrm{HL}$, et al. Domain binding and isotype dictate the activity of anti-human 0X40 antibodies. Journal for ImmunoTherapy of Cancer 2020;8:e001557. doi:10.1136/jitc-2020-001557

JEW and MSC contributed equally.

Accepted 24 November 2020

Check for updates

(C) Author(s) (or their employer(s)) 2020. Re-use permitted under CC BY. Published by BMJ.

${ }^{1}$ Antibody and Vaccine Group, Centre for Cancer Immunology, Cancer Sciences Unit, Faculty of Medicine, University of Southampton, Southampton, UK ${ }^{2}$ Preclinical Research, Biolnvent International AB, Lund, Sweden

Correspondence to Professor Mark S Cragg; msc@soton.ac.uk

\section{ABSTRACT}

Background Previous data suggests that anti-0X40 mAb can elicit anti-tumor effects in mice through deletion of Tregs. However, 0X40 also has powerful costimulatory effects on T cells which could evoke therapeutic responses. Human trials with anti-0X40 antibodies have shown that these entities are well tolerated but to date have delivered disappointing clinical responses, indicating that the rules for the optimal use of anti-human OX40 (h0X40) antibodies is not yet fully understood. Changes to timing and dosages may lead to improved outcomes; however, here we focus on addressing the role of agonism versus depleting activity in determining therapeutic outcomes. We investigated a novel panel of anti-h0X40 $\mathrm{mAb}$ to understand how these reagents and mechanisms may be optimized for therapeutic benefit.

Methods This study examines the binding activity and in vitro activity of a panel of anti-h0X40 antibodies. They were further evaluated in several in vivo models to address how isotype and epitope determine mechanism of action and efficacy of anti-h0X40 mAb.

Results Binding analysis revealed the antibodies to be high affinity, with epitopes spanning all four cysteine-rich domains of the OX40 extracellular domain. In vivo analysis showed that their activities relate directly to two key properties: (1) isotype-with mlgG1 mAb evoking receptor agonism and CD8+ T-cell expansion and mlgG2a mAb evoking deletion of Treg and (2) epitope-with membraneproximal mAb delivering more powerful agonism. Intriguingly, both isotypes acted therapeutically in tumor models by engaging these different mechanisms. Conclusion These findings highlight the significant impact of isotype and epitope on the modulation of anti-h0X40 mAb therapy, and indicate that CD8+ T-cell expansion or Treg depletion might be preferred according to the composition of different tumors. As many of the current clinical trials using 0X40 antibodies are now using combination therapies, this understanding of how to manipulate therapeutic activity will be vital in directing new combinations that are more likely to improve efficacy and clinical outcomes.

\section{INTRODUCTION}

The use of immunomodulating monoclonal antibodies (mAb) to generate anti-tumor immune responses offers an exciting approach to cancer immunotherapy. $\mathrm{mAb}$ against immune checkpoint inhibitors such as ipilimumab and nivolumab, which target the co-inhibitory receptors CTLA-4 and PD-1, respectively, pioneered this approach and have demonstrated success in treating a number of previously untreatable cancers. ${ }^{2}$ However, many patients do not respond to these reagents and additional therapeutic strategies are required. Agonistic $\mathrm{mAb}$ targeting co-stimulatory receptors have emerged as targets for clinical development, in particular, tumor necrosis factor receptors (TNFR) superfamily members such as CD40, ${ }^{3}$ $4-1 \mathrm{BB},{ }^{4}$ and OX40. ${ }^{5-7}$ However, Freeman et $a l^{8}$ identified an intratumoral Treg signature which included TNFR family members with the hypothesis that they could be targeted instead by depleting antibodies in order to generate therapy. TNFR family members are typically characterized by an extracellular domain (ECD) consisting of several cysteinerich domains (CRDs) which allow for binding of their respective trimeric ligands leading to receptor clustering and downstream signaling. ${ }^{9}$ mAb targeting such receptors have been shown to depend on their interaction with the inhibitory Fc $\gamma \mathrm{R}$ (Fc $\gamma \mathrm{RIIB})$ to generate sufficient cross-linking and resultant agonistic activity. ${ }^{1011}$ More recently, the ability of a number of TNFR mAb to cause deletion of Tregs via engagement of activatory $\mathrm{Fc} \gamma \mathrm{R}$ has been demonstrated. ${ }^{12} 13$ The anti-mouse OX40 mAb, OX86, has previously been shown to enhance effector T-cell proliferation and survival leading to successful therapeutic outcomes in pre-clinical models. ${ }^{5} 14$ Recently, it has also been demonstrated to be capable of deleting Tregs in an activatory Fc $\gamma R$-dependent manner. ${ }^{12}$ This effect was directly influenced by isotype, with mIgG2a 
showing greater depleting capacity than the native $\mathrm{IgG1}$ isotype. Interestingly, Tregs were preferentially deleted over effector $\mathrm{T}$ cells which correlated with mOX40 expression on these cells. ${ }^{12}$

Work on several TNFRs has further highlighted the importance of the region targeted by the antibody in influencing the type and strength of effector function. ${ }^{15-17}$ For anti-CD40 mAb, the membrane distal CRD1-binding $\mathrm{mAb}$ were shown to be strong agonists of CD40 with membrane proximal mAb less potent. ${ }^{16}$ Furthermore, mAb binding CRD2-4 blocked CD40L and were potent antagonists. Additionally, anti-4-1BB mAb, which bound membrane proximal domains, engaged in more effective complement-dependent cytotoxicity and antibodydependent cellular cytotoxicity killing mechanisms with antibody-dependent cellular phagocytosis less affected. ${ }^{15}$ Moreover, Zhang et $a l^{17}$ reported that mAb binding to mouse (m)OX40, which blocked ligand binding and bound CRD2, or bound at the membrane proximal domain (CRD4), provide stronger agonistic and antitumor activity than mAb binding CRD1 and 3. These results differed from those seen for hCD40, highlighting that the functional effects of mAb domain binding are likely to require assessment for each of the TNFR family members and validation for each species.

Given these discrepancies, we explored the optimal domain binding and isotype for a novel panel of antihuman (h)OX40 mAb which collectively bound to all four CRDs of the ECD of hOX40. We evaluated their function in vitro and in vivo as both mIgG1 and mIgG2a isotypes. Using a novel hOX40 knock-in (KI) mouse, we found that mIgG1 mAb were agonistic and engendered memory responses, whereas mIgG2a mAb had depleting activity with poorer memory recall responses. The strength of these effector functions appeared to correlate with domain binding; those $\mathrm{mAb}$ which bound to the most membrane proximal domain (CRD4) which did not block ligand binding, showed the strongest agonistic activity as mIgG1 as well as the most potent depleting activity as mIgG2a. This data highlights how mAb to different TNFR (and different species) exhibit different requirements in relation to optimal domain binding and effector function, and indicate how more active anti-hOX $40 \mathrm{mAb}$ might be developed.

\section{RESULTS \\ h0X40KI mice express h0X40 and develop normally}

To investigate the immunotherapeutic potential of a new panel of anti-hOX $40 \mathrm{mAb}$, we generated a new KI mouse, designed to express hOX40 ECD and mOX40 transmembrane and intracellular domains (online supplemental figure 1A). PCR confirmed integration of the construct and identified wildtype (WT), hOX40 $\mathrm{KI}^{+/-}$ and $\mathrm{hOX} 40 \mathrm{KI}^{+/+}$mice (online supplemental figure 1B). Analysis of OX40 surface expression (mouse and human) on resting splenocyte T cells from $\mathrm{WT}, \mathrm{hOX} 40 \mathrm{KI}^{+/-}$and hOX $40 \mathrm{KI}^{+/+}$mice confirmed that the chimeric receptor was expressed at the cell surface on relevant cell types (figure $1 \mathrm{~A}$ and $\mathrm{B}$ ) and in a gene dose-dependent manner (online supplemental figure 1C). Expression of OX40 in all three genotypes was largely restricted to T-cell lineages (figure 1A and B and online supplemental figure 1D). In line with previous reports, ${ }^{12}{ }^{18-20}$ a hierarchal expression pattern among the T-cell subsets was observed in all genotypes, with expression highest on Tregs followed by CD4+ effectors, with limited expression on resting CD8+ T cells (figure $1 \mathrm{~A}$ and $\mathrm{B}$ ). To address whether the hOX40 $\mathrm{KI}^{+/+}$ mice represented a functional model to study the OX40L:OX40 axis, we performed surface plasmon resonance (SPR) analysis of mOX40L and hOX40L binding to hOX40 (online supplemental figure 1E). Both mOX40L and hOX40L bound similarly to hOX40, in agreement with earlier studies, showing that mOX40L engages the same domains on hOX40 as hOX40L. ${ }^{21}$ Furthermore, OX40 knock-out (KO) mice are reported to have a subtle defect in Treg numbers (reviewed in $^{22}$ ). However expression of the chimeric receptor did not affect normal immune cell development (online supplemental figure $1 F$ ), further indicating that OX40L:OX40 signaling axis is intact in these hOX40KI mice.

Consistent with previous findings, ${ }^{23}$ activated splenocytes isolated from WT, hOX $40 \mathrm{KI}^{+/-}$and hOX40 $\mathrm{KI}^{+/+}$ mice showed peak OX40 expression between 24 and 48 hours post activation (figure 1C). The kinetics of expression of hOX40 on hOX40KI ${ }^{+/-}$and hOX $40 \mathrm{KI}^{+/+}$ splenocytes correlated with that of hOX40 on activated human peripheral blood mononuclear cells (hPBMCs) (figure 1D). A hierarchy of expression was also observed in both activated splenocytes and hPBMCs with greatest expression detectable on Tregs (figure $1 \mathrm{C}$ and $\mathrm{D}$ ). Samples taken from human cancer patients also showed the same pattern (Tregs $>$ CD4+effectors $>$ CD8+T cells) with highest OX40 expression on $\mathrm{T}$ cells isolated from tumor sites (figure 1E). Collectively, these results validated the use of the hOX40KI model to assess anti-hOX 40 mAb.

\section{Generation and characterization of a panel of anti-h0X40 mAb} A panel of seven anti-hOX $40 \mathrm{mAb}$ were subsequently generated by conventional hybridoma technology and characterized. All mAb displayed a high affinity for hOX40 (KD values between $10^{-9}$ and $10^{-10} \mathrm{M}$ ) as determined by SPR (online supplemental figure $2 \mathrm{~A}$ ) and did not bind mOX40 (online supplemental figure $2 \mathrm{~B}$ and $\mathrm{C}$ ). The crystal structure of hOX40 indicates four CRDs. ${ }^{21}$ To determine which of these the anti-hOX $40 \mathrm{mAb}$ bound, hOX40 domain mutants were generated, lacking CRD1, CRD1+2 or CRD1, 2+3 (figure 2A). A hCD20 epitope tag and mOX40 CRD3 domain were added to the final construct to stabilize its expression. Across the panel, at least one antibody bound to each of the different domains (figure 2B and online supplemental figure 2D). Crossblocking experiments showed that anti-hOX40 mAb which bound to the same domain blocked the binding of one another, whereas mAb binding to different CRDs 


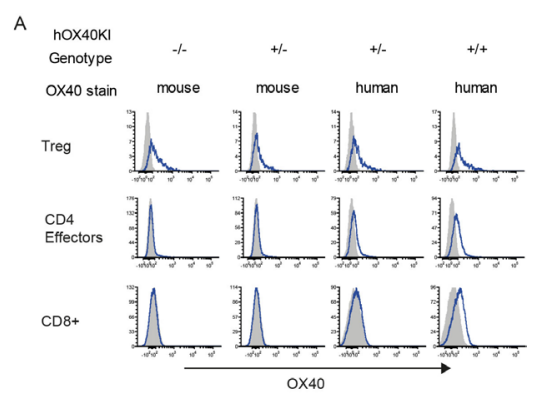

B
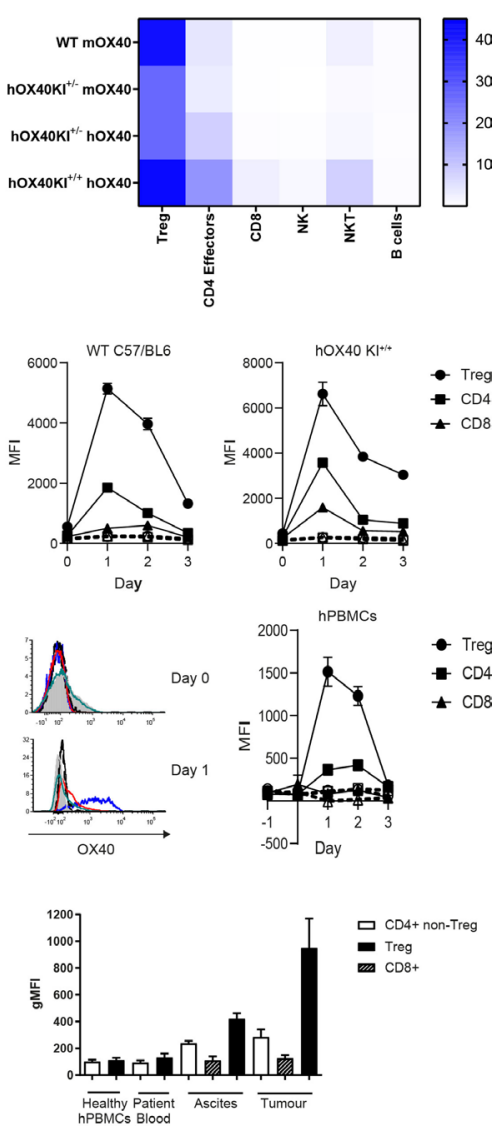

Figure 1 hOX40KI mice express hOX40 in a hierachial manner. (A). Expression of OX40 (mouse (m) and human (h) blue line) compared with isotype control (shaded histogram) on Treg (top row), CD4+ effectors (middle row) and CD8+ T cells (bottom row). Representative plots shown. (B) Heat map summarizing OX40 expression as a percentage on resting mouse splenocytes $(n=4)$. (C) Expression of mOX40 (left panel) and hOX40 (right panel) on splenocytes from WT or hOX40KI mice activated with $\alpha C D 3$ and $\alpha C D 28(n=3)$. Isotype controls showed as dashed lines. (D) Expression of hOX40 on hPBMCs activated with $\alpha C D 3$ and $\alpha C D 28$. Histograms show hOX40 expression on Tregs (blue line, isotype control black line), CD4+ (red line, isotype control black dashed line) and CD8+ (green line, isotype control gray filled histogram) from a representative donor on Day 0 (top panel) and Day 1 (bottom panel). Line graph (right panel) shows average expression, isotype controls shown as dashed lines $(n=3)$. (E) hOX40 expression on CD4+ effectorT cells (white bars), CD8+ T cells (hatched bars) or Tregs (black bars) isolated from healthy donors or blood, ascites and tumor from cancer patients ( $n=4-16)$. MFI, mean fluorescence intensity; gMFI, geoMean fluorescence intensity; hPBMC, human peripheral blood mononuclear cells; KI, knock-in; WT, wildtype. could bind simultaneously (figure 2C). The domain binding for each antibody is summarized in figure 2D. Finally, SPR analysis revealed that only mAb binding to CRD4 were able to bind in the presence of the ligand (figure 2E and online supplemental figure 2E), indicating ligand binding blocks binding of $\mathrm{mAb}$ that recognize epitopes in CRD1-3.

Antibodies were then assessed for their ability to augment suboptimal anti-CD3-mediated proliferation of hPBMC. All of the mIgG1 mAb increased proliferation of CD8+ T cells (figure 2F) whereas mIgG2a mAb reduced proliferation. Differences between mIgG1 and mIgG2a isotypes have previously been reported for other TNFR family members ${ }^{24}$ and so we class-switched the mAb so that both mIgG1 and mIgG2a isotypes were available. A similar trend was seen in hOX40KI splenocytes stimulated with anti-hOX40 mAbs with mIgG1 mAb showing a trend towards an increase in proliferation while mIgG2a $\mathrm{mAb}$ showed a clear reduction in proliferation (online supplemental figure 3).

\section{Anti-h0X40 mlgG1 mAb are agonistic in vivo}

To investigate the ability of the anti-hOX $40 \mathrm{mAb}$ to cause antigen-specific CD8+ T-cell expansion in vivo, we used the OT-I model whereby antigen-specific $\mathrm{T}$ cells are transferred into naive recipients. hOX $40 \mathrm{KI}^{+/-}$OT-I $\mathrm{T}$ cells, which recognize the $\mathrm{OVA}_{257-264} / \mathrm{H}-2 \mathrm{~K}^{\mathrm{b}}$ complex, were adoptively transferred into hOX40 $\mathrm{KI}^{+/+}$mice before vaccination with OVA and administration of anti-hOX40 $\mathrm{mAb}$ as either mIgG1 or mIgG2a (figure 3A). Both mIgG1 and mIgG2a anti-hOX40 mAb expanded antigen-specific CD8+ OT-I T cells in blood compared with OVA alone and to a similar extent with the exception of SAP 28-2 which was notably weaker as a mIgG2a (figure 3B and online supplemental figure 4A). Despite reaching similar frequencies at the peak of the primary response, on re-challenge with SIINFEKL peptide alone, a significantly smaller recall response was seen in mice that had received mIgG2a antibodies (figure 3B and online supplemental figure 4B). Given the time between antibody administration and re-challenge with SIINFEKL peptide, it is likely this lack of recall reflects responses initiated during the priming stage as opposed to any effects of $\mathrm{mAb}$ persisting from the initial challenge. The frequency of OT-I cells prerecall in mIgG1-treated mice strongly correlated with the strength of the recall peak (online supplemental figure $4 \mathrm{C}$ ); however due to the lack of a recall response seen in the mIgG2a it is not possible to determine if this is also the case for the mIgG2a isotype. These data suggest that the number of OT-I cells present before re-challenge is a key determining factor for the strength of the recall response and that mIgG1 and mIgG2a mAb deliver signals during the primary response which results in different resting memory populations.

To understand this difference in memory response, OT-I $\mathrm{T}$ cells in the blood were phenotyped during the primary and memory stages. Analysis of CD127 and KLRG1 expression during the primary response can identify 


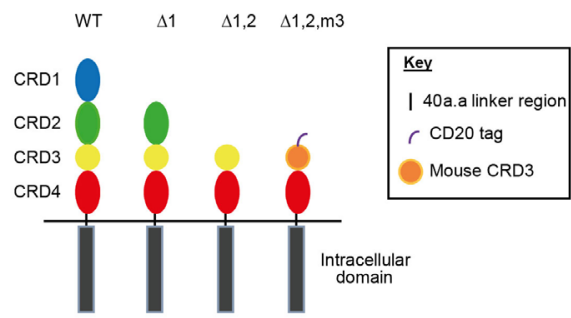

C.

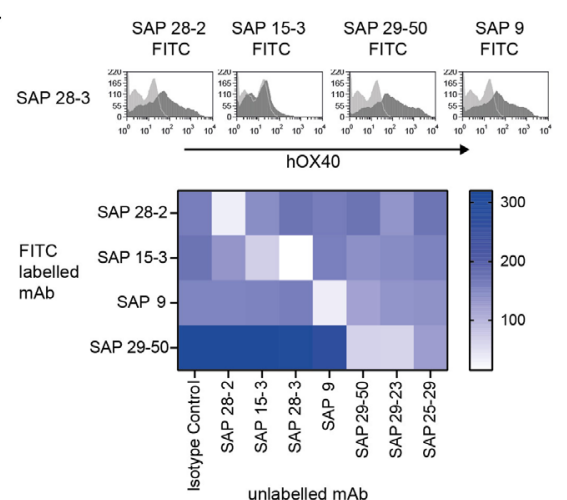

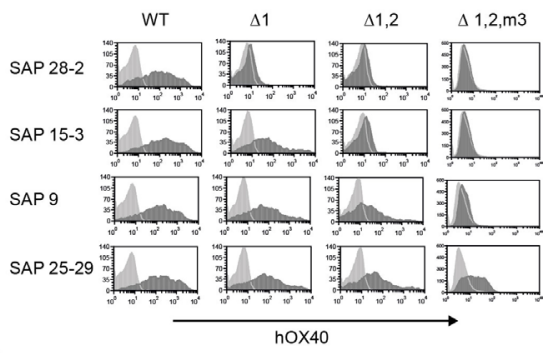

D.

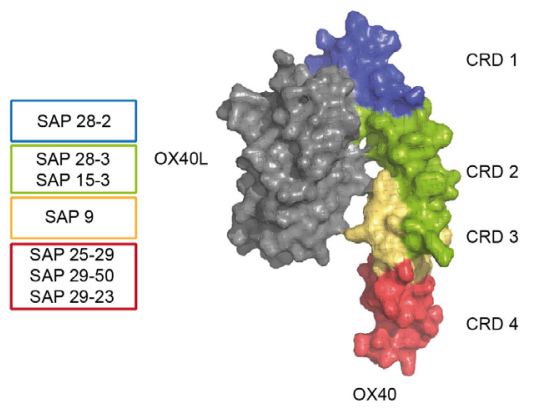

E.

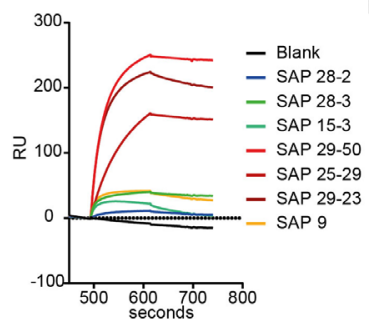

F.

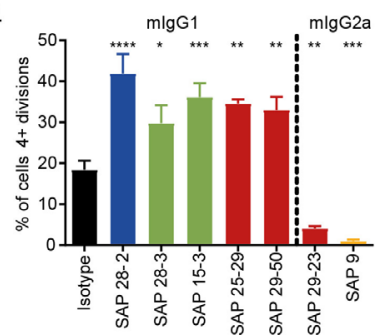

Figure 2 Characterization of a panel of anti-hOX40 mAb. (A) Schematic of the WT and domain mutant hOX40 constructs generated. CRD3 from mOX40 was used to stabilize the human CRD4 construct. (B) Binding of anti-hOX40 mAb to domain constructs detected by a PE-labeled secondary $\mathrm{Fab}_{2}$ fragments. Representative histograms show hOX40 mAb binding (dark gray histogram) compared with an isotype control (light gray histogram). (C) Representative histograms show anti-hOX40 FITClabeled mAb binding (dark gray histogram) in relation to an isotype control (light gray histogram) after binding of unlabeled anti-hOX40 mAb. The heat map shows MFI of FITC-labeled antibody binding in the presence of unlabeled antibodies with the absence of color indicating blocking. (D) Diagram summarizing antibody binding domains in relation to the crystal structure of the OX40:OX40L complex. (E) Surface plasmon resonance analysis of anti-hOX40 mAb binding to hOX40-hFc in the presence of hOX40L-His fusion protein. (F) Proliferation of hCD8+ T cells within PBMC cultures in response to sub-optimal anti-CD3 and anti-hOX40 mAb stimulation (representative of four individual donors). Mean $\pm S E M{ }^{* \star *} p<0.0001,{ }^{\star \star *} p<0.001,{ }^{* \star} p<0.01,{ }^{*} p<0.05$, Dunnett's multiple comparison test. PE, Phycoerythrin; MFI, mean fluorescence intensity; CRD, cysteine-rich domain; FITC, fluorescein isothiocyanate; mAb, monoclonal antibody; PBMC, peripheral blood mononuclear cells; WT, wildtype.

short-lived effector cells (SLECs-CD127- KLRG1+) and memory precursor cells (MPECs-CD127+ KLRG1-). ${ }^{26}$ The frequency of MPECs was higher in the mIgG1 groups at Day 18 (figure 3C). While frequencies of SLECs in the blood was similar between isotypes (online supplemental figure 4D) granzyme B production was higher in SAP 9 and 25-29 mIgG2a-treated mice compared with mIgG1-treated mice (figure 3C). Additionally, these data indicated that there may be a domain-related trend in granzyme $\mathrm{B}$ production in those mice receiving $\mathrm{mIgG2a}$ $\mathrm{mAb}$, with the following hierarchy; CRD4 binding $\mathrm{mAb}$ (SAP 25-29) >CRD3 (SAP 9) >CRD2 (SAP 15-3) and CRD1 (SAP 28-2). In infection models, the relative frequencies of each subpopulation (SLECs versus MPECs) in the primary response does not always correlate with the accumulation of CD8+ cells during a recall response. ${ }^{26-28} \mathrm{We}$ therefore expanded our analysis to CXCR3 and CD43, shown to define three distinct populations of memory cells with a hierarchy of recall response $\left(\mathrm{CXCR} 3^{\text {hi }} \mathrm{CD} 43^{\text {lo }}\right.$ $>$ CXCR $3{ }^{\text {hi }} \mathrm{CD} 43^{\text {hi }}>$ CXCR $\left.3{ }^{\text {lo }} \mathrm{CD} 43^{\text {lo }}\right) .{ }^{29}{ }^{30}$ Mice that had been treated with mIgG1 anti-hOX40 mAb gave rise to a higher frequency of CXCR $3{ }^{\text {hi }} \mathrm{CD} 43^{\text {lo }}$ and CXCR $3{ }^{\text {hi }} \mathrm{CD} 43^{\text {hi }}$ cells in comparison to mice that had been treated with mIgG2a mAb (figure 3D and online supplemental figure $4 \mathrm{E})$. This effect was most strongly seen with the SAP 25-29 CRD4-binding antibody (figure 3D and online supplemental figure $4 \mathrm{E}$ ). This difference in frequencies between mIgG1-treated and mIgG2a-treated mice, while slight during the contraction phase (D18), became more evident during the resting memory period prior to re-challenge (D53). Immediately following re-challenge with SIINFEKL peptide (D74), both mIgG1 and mIgG2a 
A.
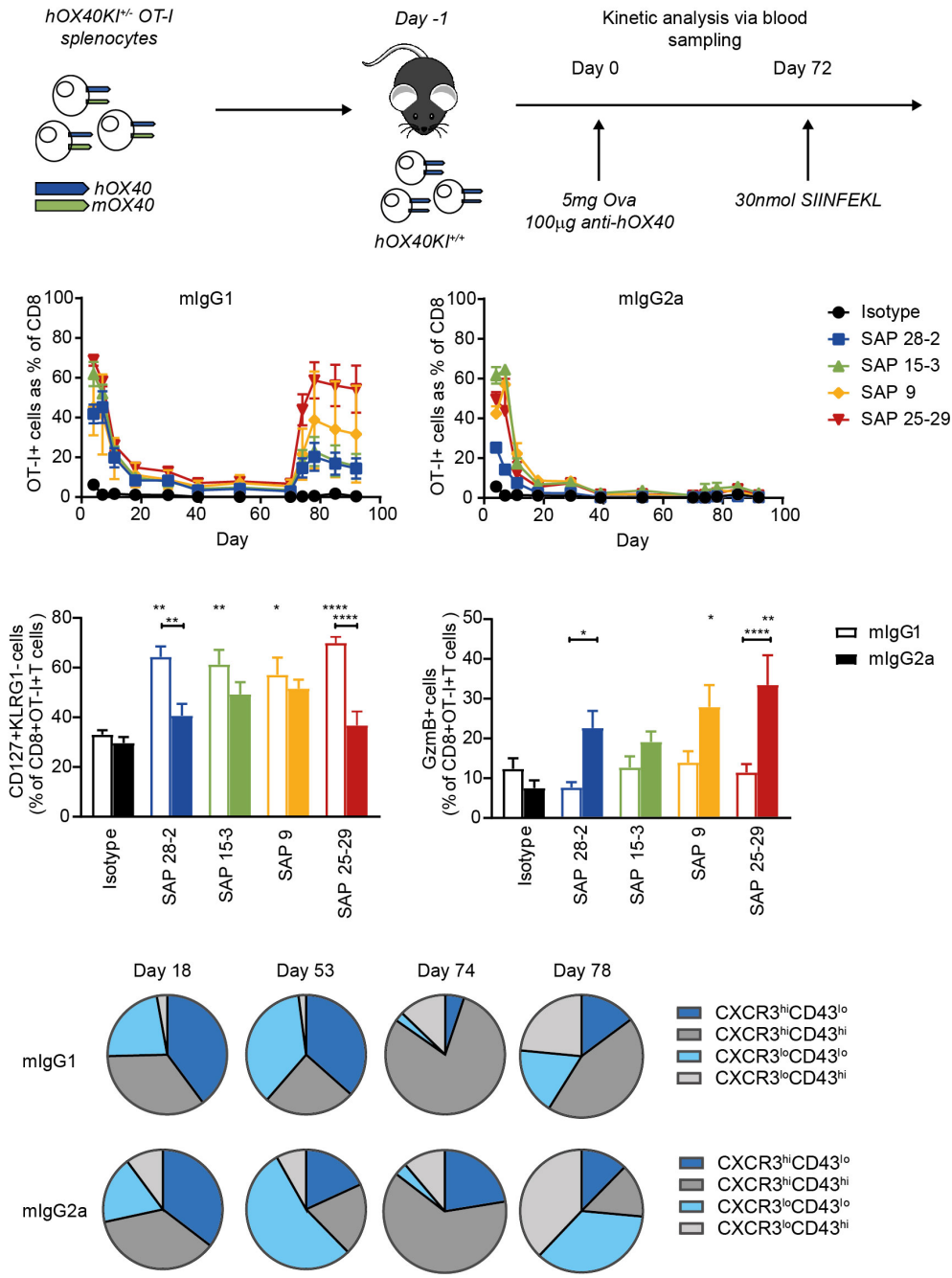

E.
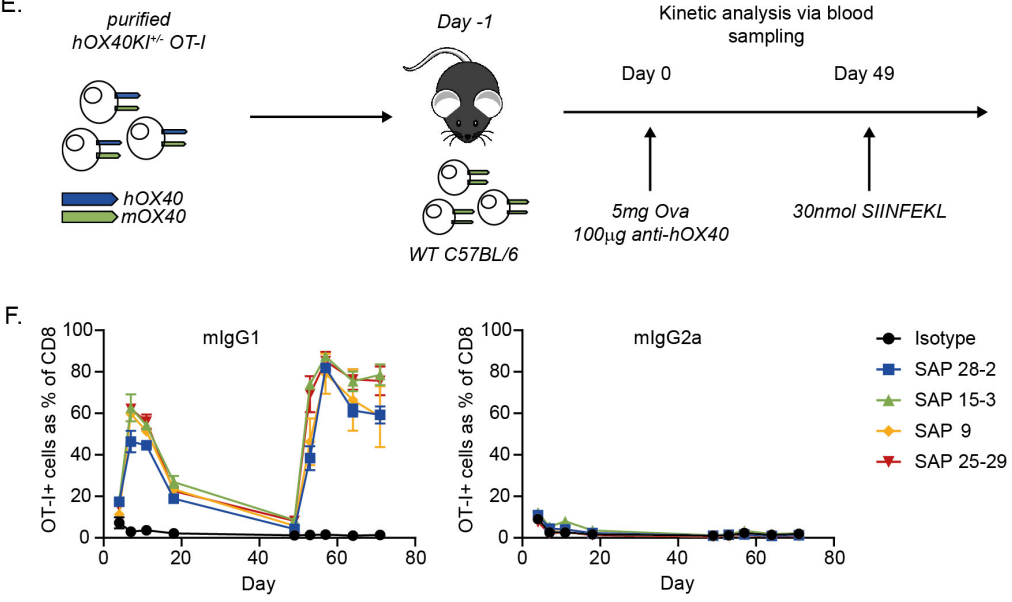

Figure 3 Anti-hOX40 mlgG1 act agonistically in vivo. (A) Schematic of the OT-I model used in B-D. $1 \times 10^{5} \mathrm{hOX}^{\circ} \mathrm{KI}^{+/-}$OTI cells were transferred into hOX40KI ${ }^{+/+}$recipients. Mice were challenged with $5 \mathrm{mg}$ OVA and $100 \mu \mathrm{g}$ of antibody. (B). Kinetic analysis of OT-I expansion in response to anti-hOX40 mlgG1 mAb (left panel) or mlgG2a mAb (right panel) ( $n=4$ representative of two independent experiments). (C) Analysis of memory and effector phenotyping of OT-I T cells in blood at Day 18. MPECsCD127+ KLRG1 - (left panel) and granzyme B+ OT-I T cells (right panel) ( $n=4$ isotype controls and $n=8$ all treatment groups, pooled from two independent experiments). (D) CXCR3/CD43 profiling of OT-I T cells in blood at time points indicated in response to SAP 25-29 stimulation $(n=4)$. (E) Schematic of the OT-I model used in $\mathrm{F}$. $1 \times 10^{5} \mathrm{hOX} 40 \mathrm{KI}^{+/-}$OT-I were transferred into WT C57BL/6 recipients. Mice were challenged with $5 \mathrm{mg}$ OVA and $100 \mu \mathrm{g}$ of antibody. (F) Kinetic analysis of OT-I expansion in response to anti-hOX40 mlgG1 mAb (left panel) or mlgG2a mAb (right panel) ( $\mathrm{n}=4$ representative of two independent experiments). Mean $\pm S E M{ }^{* \star * \star} p<0.0001,{ }^{* \star} p<0.01{ }^{\star} \mathrm{p}<0.05$, Sidak's multiple comparison test. mAb, monoclonal antibody; MPEC, memory precursor cells; WT, wildtype. 
groups displayed expansion of cells with high proliferative capacity, but further into the memory response (D78), the higher prevalence of cells with greater proliferative capacity in mIgG1-treated mice was re-established (figure 3D). Furthermore, mice treated with mIgG2a anti-hOX40 mAb had a higher frequency of effector-like memory cells $\left(\mathrm{CXCR} 33^{\text {lo }} \mathrm{CD} 43^{\text {lo }}\right)$ in the resting memory phase (D53) (figure 3D). Again, this contrast between mIgG1-treated and mIgG2a-treated mice was re-established from D78 following re-challenge. These data indicate, that in our OT-I transfer model, the choice of isotype significantly impacts the development of a robust memory response.

These results also highlighted a discrepancy in the effect of the mIgG2a anti-hOX40 mAb between in vitro and in vivo experiments. mIgG2a anti-hOX $40 \mathrm{mAb}$ caused an inhibition of proliferation in vitro (figure $2 \mathrm{~F}$ ), whereas T-cell expansion was seen in vivo (figure $3 \mathrm{~B}$ ). Therefore, hOX40 $\mathrm{KI}^{+/-}$CD8+ OT-I T cells were purified and transferred into WT C57BL/6 mice to see if both mIgG1 and mIgG2a isotypes were capable of acting directly on CD8+ $\mathrm{T}$ cells (figure $3 \mathrm{E}$ ). While mIgG1 mAb were able to drive a similar expansion as before, mIgG2a mAb had no effect (figure $3 \mathrm{~F}$ ). These results indicate that the mIgG2a mAb act indirectly, through non-CD8 $+\mathrm{T}$ cells, to facilitate OT-I expansion in contrast to the ability of mIgG1 to act directly on the OT-I T cells.

\section{Anti-h0X40 mlgG2a mAb deplete h0X40 expressing cells}

We hypothesized that the indirect effect of mIgG2a mAb might involve deletion of Treg. ${ }^{24}$ Depletion of Tregs has previously been shown to allow greater expansion of OT-I and OVA responses. ${ }^{31-33}$ Therefore, to address this hypothesis, spleens were harvested from hOX $40 \mathrm{KI}^{+/+}$mice 4 days after treatment with either anti-hOX $40 \mathrm{mIgG1}$ or mIgG2a mAb (figure 4A). Numeration of different T-cell subsets in the spleen showed that the mIgG2a mAb were able to significantly reduce the Treg population and to a lesser extent CD4+ effectors whereas mIgG1-treated mice evoked expansion of T-cell populations (CD8+ and OT-I) (figure 4B and online supplemental figure 5B). The relative deletion of T-cell populations in the mIgG2a-treated mice correlated with the amount of hOX40 surface expression seen following activation in vitro (figure 1C) as the most significant difference was seen in the Treg population followed by CD4+ effectors. The CD8:Treg ratio was unchanged following treatment with the mIgG1 mAb; however, SAP 25-29 mIgG2a produced a significant increase in the CD8:Treg ratio a with a trend towards an increase also seen for SAP 15-3 mIgG2a and SAP 9 mIgG2a (figure 4B). Interestingly, SAP $9 \mathrm{mIgG} 2 \mathrm{a}$ also showed a decrease in inter-sample variability when looking at OT-I cell numbers and a trend towards a reduction in CD8+ T-cell numbers, indicating superior deletion capacity compared with other mAb. Therefore, we assessed the ability of SAP 9 to delete human Tregs in vivo. Unactivated human Treg do not typically express appreciable OX40 levels (figure 1D, ${ }^{34}$ ), so a NOD/Shi-scid/IL-2R $\gamma^{\text {null }}$
(NOG): PBMC transfer model was used whereby hPBMCs are first activated through a xenoresponsive graft versus host response before being transferred into a new NOD scid gamma (NSG) recipient mouse where deletion could then be assessed. The transferred Treg upregulate hOX40 to levels similar to those observed within tumors and significantly above the levels of CD8+ T cells (online supplemental figure 5B). Using this approach, SAP 9 hIgG1 was shown to be capable of deleting human Tregs at least as well as the clinically-approved Treg deleting antiCTLA-4 mAb Yervoy (figure 4D); thereby augmenting the CD8:Treg ratio (figure 4E). Furthermore, when using a humanized version of SAP 9 hIgG1, significant depletion of human Tregs was observed (figure 4F), alongside a significant improvement in the CD8:Treg ratio (figure 4G), unlike CAMPATH-1 which deleted all $\mathrm{T}$ cells. These data indicate that with the correct isotype and significant expression of hOX40, anti-hOX40 antibodies are capable of specifically depleting Tregs and improving CD8:Treg ratios in vivo.

\section{Extent of isotype activity correlates with 0X40 domain specificity}

Throughout the data detailed above, it became apparent that the strength of deletion/agonism was associated with the domain of hOX 40 bound by the various $\mathrm{mAb}$. To assess this further, we grouped our results into membrane distal $(\mathrm{CRD} 1+2)$ and membrane proximal (CRD3+4) binding $\mathrm{mAb}$ (figure $5 \mathrm{~A}$ and $\mathrm{B}$ ). We also assessed groups reflecting those able (CRD4) or unable to bind in the presence of ligand $(\mathrm{CRD} 1+2+3)$ to see if there was a correlation with ligand competition (figure $5 \mathrm{C}$ and $\mathrm{D}$ ). The strength of agonism seen with the mIgG1 mAb was highest for membrane-proximal binding $\mathrm{mAb}$ for both expansion of OT-I cells and Tregs (figure 5A). In contrast, with the mIgG2a isotype. only depletion of Tregs correlated with domain, again being greatest for membrane proximal $\mathrm{mAb}$ (figure $5 \mathrm{~B}$ ). Likewise, $\mathrm{mAb}$ binding outside of the ligand-binding domain (ie, CRD4) displayed the highest level of mIgG1-mediated agonism (figure 5C). Interestingly, the ability of mIgG2a mAb to deplete Tregs did not significantly correlate with binding to CRD4 (figure 5D).

\section{Ability of h0X40 mAb to control tumor growth}

To determine the immunotherapeutic potential of the anti-hOX40 $\mathrm{mAb}$ in vivo in our hOX $40^{+/+} \mathrm{KI}$ mice, we evaluated a mAb which bound to each CRD of hOX40 as both a mIgG1 and mIgG2a isotype. hOX $40^{+/+} \mathrm{KI}$ mice were inoculated with E.G7-OVA lymphoma cells and subsequently treated with anti-hOX40 mAb once tumors had established. Anti-hOX40 mAb, as both mIgG1 and mIgG2a, were able to elicit tumor control, with mice eradicating established tumors in most treatment groups (figure 6A). With the exception of SAP 25-29, the mIgG1 $\mathrm{mAb}$ caused a higher percentage of survival than the mIgG2a mAb, although there was no obvious domain preference among the antibodies in terms of anti-tumor activity. Importantly, mice also appeared to form durable 

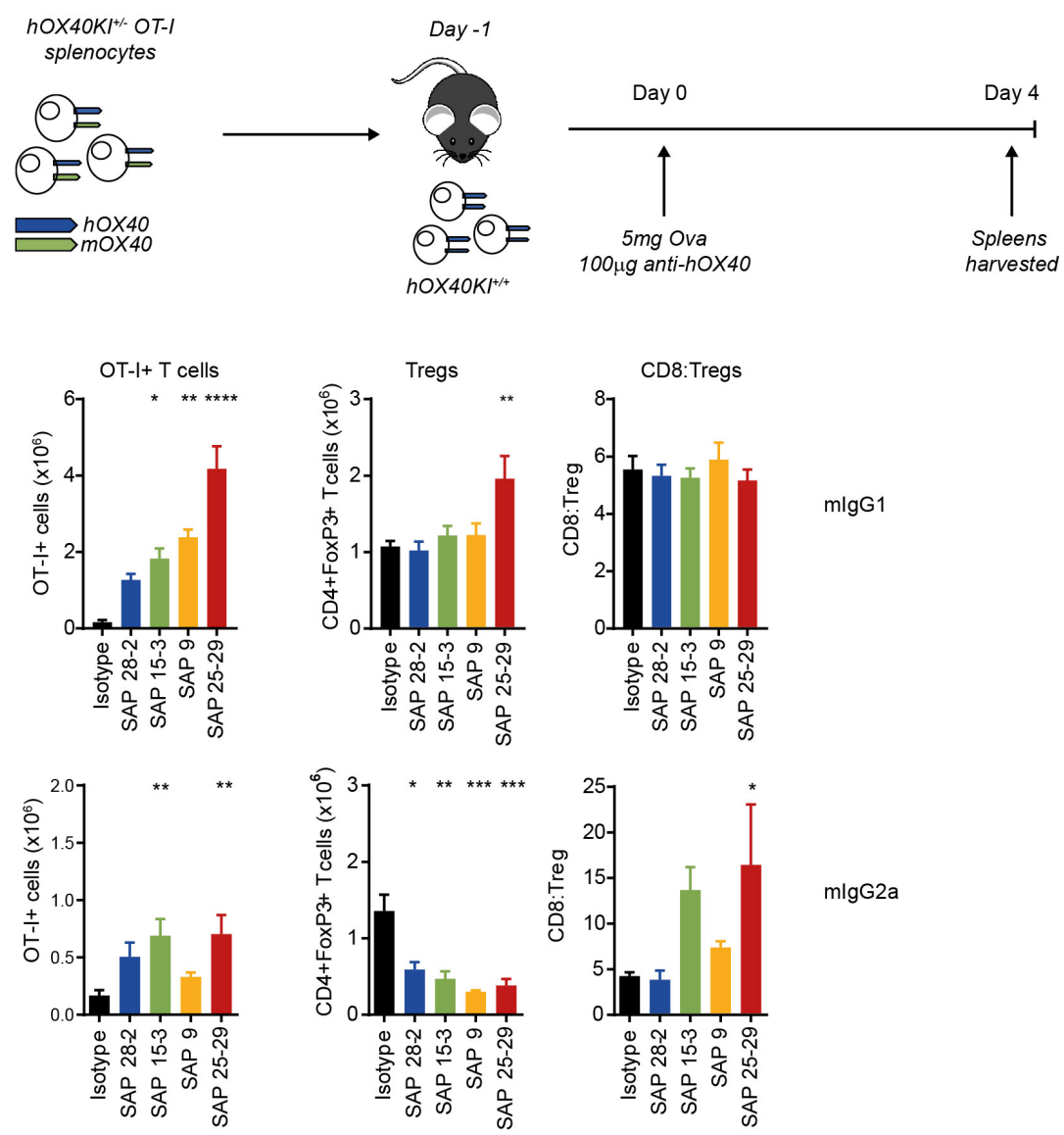

mlgG2a
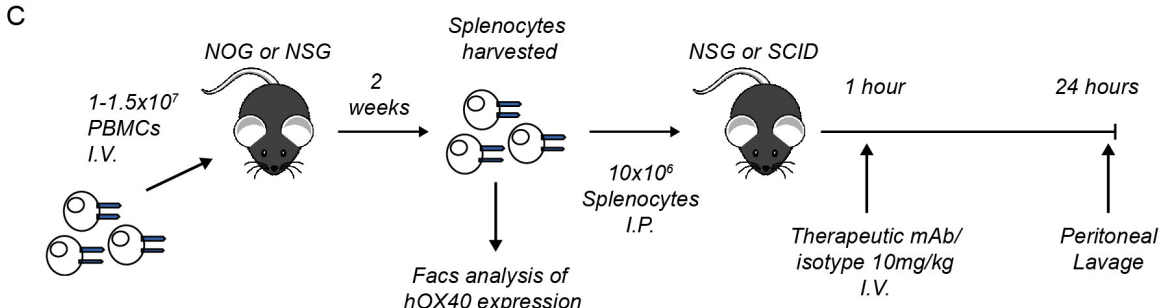

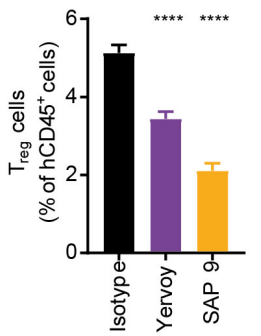

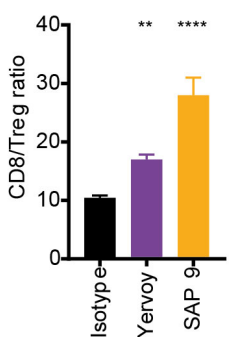

$\mathrm{F}$

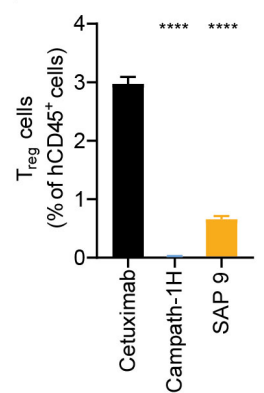

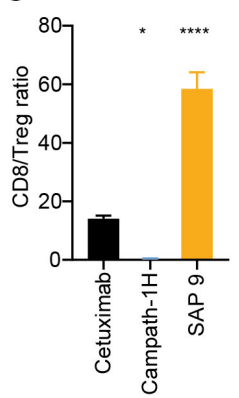

Figure 4 Anti-hOX40 mlgG2a mAb elicit Treg cell depletion in vivo. (A) Schematic of OT-I model used to assess cell depletion. (B) Assesment of Tetramer+ve (left panel), Treg cell numbers (middle panel) and CD8:Treg ratio (right panel) in response to antihOX40mAb either as a mlgG1 (top row) or mlgG2a (bottom row) ( $n=4$ SAP 28-2, $n=5$ SAP 15-3 and SAP 9, $n=6$ all remaining groups, pooled from two independent experiments). (C) Schematic of the NSG/PBMC model to assess depletion of hPBMCs. $1.5 \times 10^{7}(\mathrm{D}, \mathrm{E})$ or $1 \times 10^{7}(\mathrm{~F}, \mathrm{G})$ hPBMC were transferred into NOG (D, E) or NSG (F, G) mice. Two weeks later, splenocytes were harvested and transferred into SCID (D, E; $n=11$ SAP 9, $n=12$ Yervoy, $n=13$ isotype control pooled from two independent experiments) or NSG (F, G; $n=7$ pooled from two independent experiments) recipients which were then treated with depleting antibodies. Treg numbers $(D, F)$ and CD8:Treg ratio $(E, G)$ were determined by flow cytometry. Mean $\pm S E M$, ${ }^{* \star * *} p<0.0001$, ${ }^{\star * *} p<0.001,{ }^{* *} p<0.01,{ }^{*} p<0.05$. Sidak's $(B)$ and Tukey's ( $F$ and $G$ ) multiple comparison test. FACS, fluorescence-activated cell sorting; hPBMC, human peripheral blood mononuclear cells; mAb,monoclonal antibody; NOG,NOD/Shi-scid/IL-2R $\gamma^{\text {null; }}$ NSG, NOD scid gamma SCID, severe combined immune deficient. 


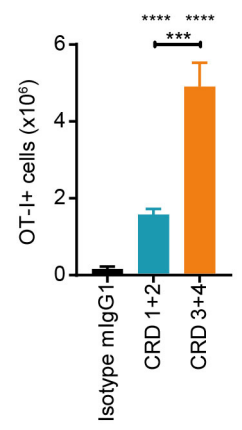

B

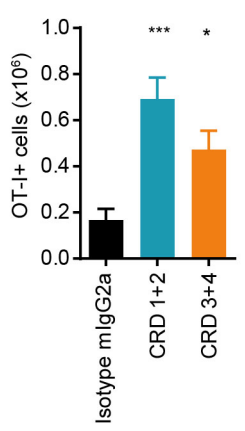

C

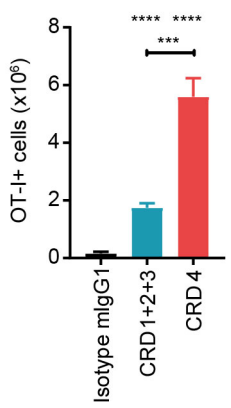

D

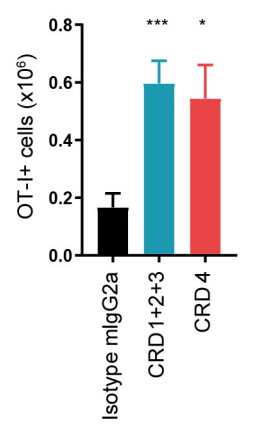

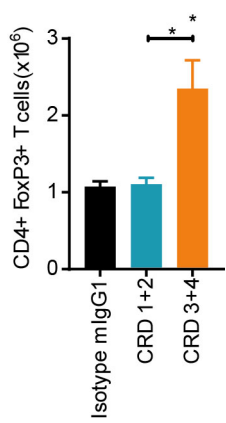
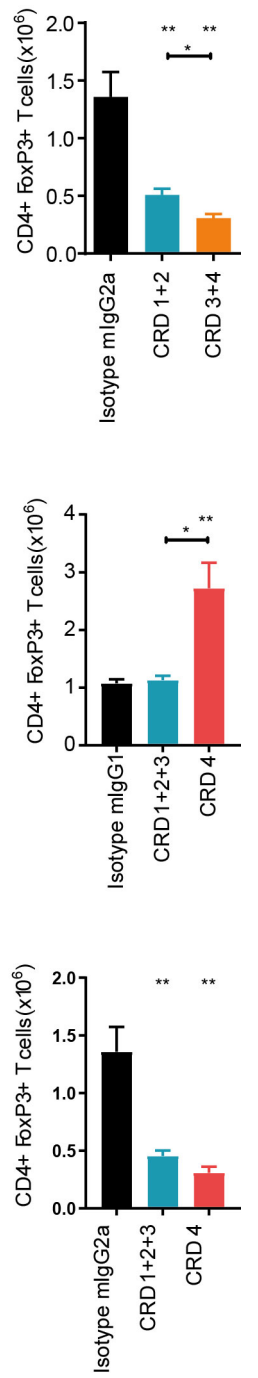

Figure 5 Anti-hOX40 mAb agonism and depleting activity are related to domain binding. Model used as in figure $4 \mathrm{~A}$ and $B$ with spleens harvested on day 4 post treatment. (A) Analysis of OT-I cell numbers (left panels) and Treg cell numbers (right panels) in response to anti-hOX40 mlgG1 mAb grouped into proximal binders $-\mathrm{CRD} 3+4$ or distal binders-CRD1+2. (B) As in A except mlgG2a mAb was used. (C) Analysis of OT-I cell numbers (left panels) and Treg cell numbers (right panels) in response to anti-hOX40 mlgG1 $\mathrm{mAb}$ grouped into those which can bind in the presence of ligand CRD4 versus those which cannot CRD1-3. (D) As in $C$, except mlgG2a mAb was used. Mean \pm SEM ${ }^{* * * *} p<0.0001$, ${ }^{\star * \star} p<0.001,{ }^{\star *} p<0.01,{ }^{\star} p<0.05$, Tukey's multiple comparison test ( $A$ and $B ; n=6-16)(C$ and $D ; n=6-19)$. CRD, cysteine-rich domain; mAb, monoclonal antibody. memory responses, as on re-challenge, no mice developed a secondary tumor (online supplemental figure 6A).

To determine the mechanism of tumor control, organs from tumor bearing mice were harvested and assessed for changes in immune inflitrate. Consistent with data from the OT-I model, the general trend seen in mIgG1-treated mice was expansion of $\mathrm{T}$ cell subsets while mIgG2a mAb caused T-cell depletion (figure 6B and $\mathrm{C}$ and online supplemental figure $6 \mathrm{~B}$ and $\mathrm{C}$ ). This effect was most prominent in the spleen but also observed within the tumor-draining lymph node (tdLN; CD4+ and Tregs). Cell numbers recovered from tumors were very small therefore it was difficult to ascertain clear trends within the tumor-infiltrating lymphocytes (TILs) (figure 6B and $\mathrm{C}$ ). A high CD8:Treg within human tumors is associated with prolonged survival ${ }^{35}$ and depletion of Tregs allows for anti-tumor immunity and rejection. ${ }^{36} 37$ Our results show that mice treated with mIgG2a anti-hOX40 tended to increase the CD8:Treg ratio within the spleen, tdLN, and tumor (figure 6C). In mIgG1-treated mice, while the spleen showed a significant reduction in the CD8:Treg ratio, in the tumor, an increased trend was observed (figure 6B). It is unclear whether this discrepancy between locations occurs as a result of different response kinetics, with priming occurring in lymphoid organs and subsequent recruitment to the tumor or perhaps direct priming within the tumor microenvironment (TME) whereby the greater antigen density leads to a more rapid response and hence increase in CD8:Treg. A domain trend could also be seen within some of the T-cell subsets; OT-I depletion in the spleen and CD4+/Treg expansion within the tdLN associated with membrane proximal domains (figure $6 \mathrm{~B}$ and $\mathrm{C}$ and online supplemental figure $6 \mathrm{~B}$ and $\mathrm{C}$ ).

The T-box transcription factors (T-bet) and eomesodermin (Eomes) cooperate to promote cytotoxic lymphocyte formation, which correlates with the upregulation of perforin and granzyme B in antigen-specific cells, ${ }^{2638}{ }^{39}$ as well as sustaining memory phenotypes. ${ }^{40}$ Thus expression of T-bet and Eomes was examined in $\mathrm{T}$ cells within the spleen, tdLN and tumor of anti-hOX40-treated mice to better understand the mechanisms involved. Both isotypes increased the T-bet+Eomes+double positive cells within the CD8+ populations (figure 6D); however, in CD4+ T cells, only mIgG1 increased this population and only in splenic populations (online supplemental figure 7A). A domain trend was also broadly observed in CD8+ populations from mIgG1-treated mice, most obviously within the spleen and tumor. CD8+ T cells within the spleen and tumor also saw an increase in granzyme B producing cells in both mIgG1-treated and mIgG2a-treated mice (online supplemental figure 7B). As with T-bet+ Eomes+ populations, CD4+ GzmB + cells were not significantly increased with either isotype (online supplemental figure 7C), thus suggesting that, in this model, we do not see a significant impact on cytotoxic CD4+ T cells. However, these results do show that all anti-hOX $40 \mathrm{mAb}$, irrespective of isotype or domain-binding region, are able to produce functional 


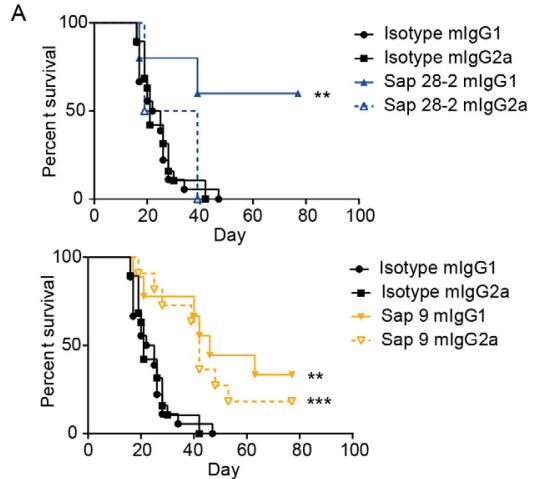

B

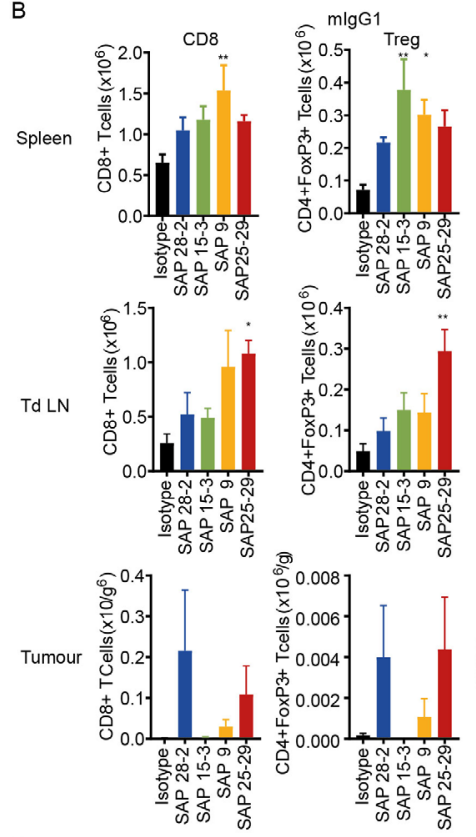

D
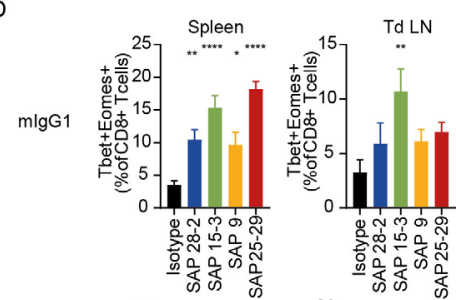
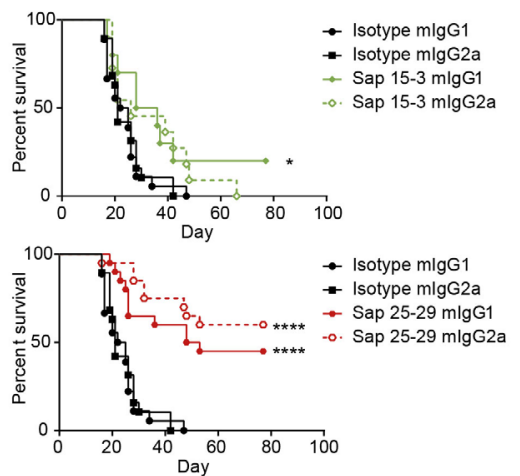

C

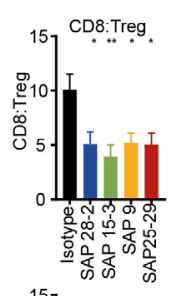

c

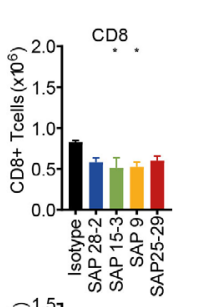

$\sigma_{0}^{m l g G 2 a}$ Treg

Isotype mlgG2a

Sap 15-3 mlgG1

Sap 15-3 mlgG2a

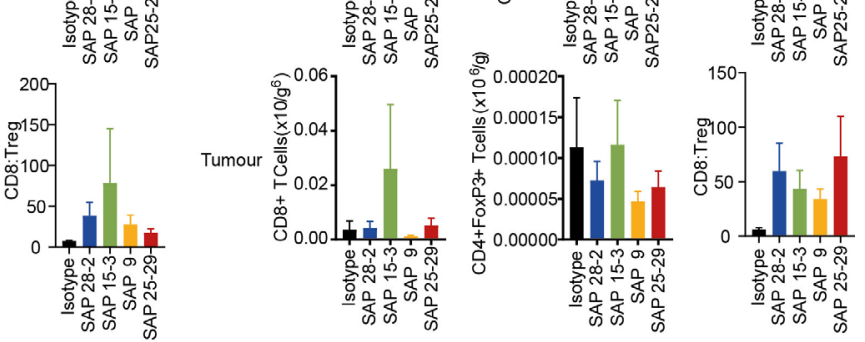

E
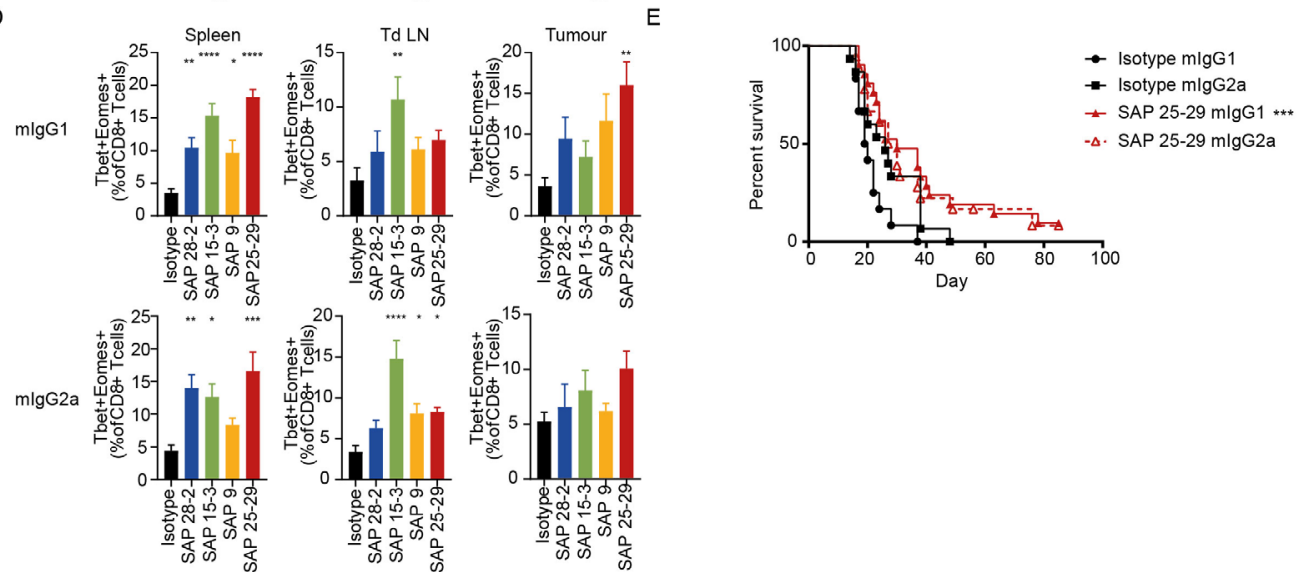

Figure 6 Anti-hOX40 mAb are therapeutic as both mlgG1 and mlgG2a. (A) Survival curves for mice challenged with E.G7 lymphoma cells $\left(0.5 \times 10^{6}\right)$ and treated with $3 \times 100 \mu \mathrm{g}$ mAb once tumors are between $5 \times 5 \mathrm{~mm}$ and $10 \times 10 \mathrm{~mm}$. Data pooled from two independent experiments ( $n=5$ SAP 28-2, $n=10$ SAP $15-3$ and SAP $9, n=20$ isotype controls and SAP 25-29). assessment of T-cell populations in spleen (top panels), tdLN (middle panels), and tumor (bottom panels) isolated 24 hours post second $\mathrm{mAb}$ dose either as a mlgG1 (B) or mlgG2a (C). $n=5$ except for SAP 28-2 mlgG2a $n=4$ for all organs and SAP15-3 and SAP 25-29 mlgG1 in tumor $n=3$ due to tumor regression, representative of two independent experiments. (D) Analysis of T-box transcription factors and eomesodermin expression in CD8+ T cells isolated from spleen (left panels), tdLN (middle panels) and tumor (right panels) from mice treated with either mlgG1 (top row) or mlgG2a (bottom row). Data pooled from two independent experiments $(n=8$ SAP $28-2$, all other groups $n=9)$. (E) Survival graphs for mice challenged with MCA-205 cells $\left(0.5 \times 10^{6}\right)$ and treated with $3 \times 100 \mu \mathrm{g}$ mAb once tumors were $5 \times 5 \mathrm{~mm}$. Data pooled from three independent experiments $(\mathrm{n}=12$ isotype mlgG1, $\mathrm{n}=15$ isotype mlgG2a, $n=18$ SAP 25-29 mlgG2a and n=21 SAP 25-29 mlgG2a). Mean \pm SEM ${ }^{\star \star \star \star *} p<0.0001,{ }^{\star \star \star} p<0.001,{ }^{* *} p<0.01,{ }^{*} p<0.05$, Logrank (Mantel-Cox) for survival graphs ( $A$ and $E$ ) and Dunnett's multiple comparison test (B to $D)$. mAb, monoclonal antibody; tdLN, tumor-draining lymph node. 
effector CD8+ T cells, as well as CD8+ T cells expressing transcription factors important for effector and memory cell formation within a tumor environment.

To determine if the ability of these mAb to evoke tumor control was consistent across tumor models we used the subcutaneous MCA-205 sarcoma. Using SAP 25-29 as a paradigm, we again saw both isotypes providing tumor control, although more limited in comparison to the E.G7 tumor model and with no isotype preference (figure 6E).

\section{DISCUSSION}

In the current study, we generated and characterized a panel of mAb-targeting hOX40. Their ability to bind throughout the four different hOX40 CRDs and expression as both mIgG1 and mIgG2a isotypes allowed investigation of the effects of both isotype and domain binding on agonistic and therapeutic potential in a newly developed hOX40 KI mouse model.

The hOX40 KI mouse model displayed an expression pattern of hOX 40 largely reflecting that seen on healthy hPBMCs and samples from ovarian cancer patients with a hierarchy of expression of Treg $>$ CD4 $+>$ CD $8+$. Nevertheless, differences were observed such that higher levels of hOX40 were seen on peripheral CD8+ T cells in the homozygous KI mouse than would be expected on hPBMC. Additionally, constitutive expression of hOX 40 was observed on peripheral Tregs in the mice, in contrast to negligible levels on resting hPBMCs. However, this expression pattern reflects that observed on Treg in TILs isolated from cancer patients and so appears a reasonable model for studies in oncology.

In the hOX40 KI mouse, mOX40L not hOX40L is present which may have led to immune defects due to the absence of OX40L:OX40 interaction. However, no overt differences in immune development or homeostasis were observed in the homozygous hOX40 KI mice. Furthermore, mOX40L has been shown to make similar contacts to hOX40 as hOX40L, ${ }^{21}$ suggesting that the OX40L:OX40 signals would be maintained in our model. It also indicates that the model is suitable to address any influences of ligand binding on the activity of the $\mathrm{mAb}$ panel. Only mAb binding to the most membrane proximal domain (CRD4), were able to bind in the presence of the natural ligand OX40L (CD252), raising the possibility that certain effector functions could be influenced by concurrent ligand binding for the CRD4-binding mAb. Although not studied directly, no overt effects appeared to be driven by the presence or absence of OX40L binding; that is, all antibodies regardless of ligand blocking were able to elicit function in an OT-I transfer model and in tumor models.

It is well known that isotype helps dictate mAb effector function due in part to differences in Fc $\gamma \mathrm{R}$ interactions, and so isotype choice is important for delivering therapeutic efficacy according to the mAb mechanism of action. ${ }^{24}{ }^{41} 42$ For TNFR family members, mIgG1 antibodies have been agonistic with engagement of Fc $\gamma$ RII and
mIgG2a being either inhibitory or with limited agonistic effects yet capable of activatory Fc $\gamma$ R-mediated target cell depletion. ${ }^{12} 182425$ Our hPBMC proliferation data added to this evidence, with hOX40 mIgG2a mAb resulting in a decrease in the percentage of proliferating $T$ cells whereas mIgG1 counterparts evoked increases in T-cell proliferation. However, this was not reflected in our hOX $40^{+/-}$ OT-I transfer studies in hOX $40^{+/+} \mathrm{KI}$ mice, where both anti-hOX40 mAb isotypes were able to cause strong expansion of OT-I cells. However, when hOX $40^{+/-}$OT-I cells were transferred into WT mice, the mIgG2a mAb were no longer able to elicit expansion of OT-I cells, unlike the mIgG1 $\mathrm{mAb}$, which retained this activity. Together, these results indicate that the mIgG1 mAb causes direct agonism on the hOX $40^{+/-}$OT-I cells, resulting in their expansion, whereas the mIgG2a mAb require hOX40 expressing non-CD8+ cells to provide expansion. Previous experiments by Ruby $e t$ $a l^{43}$ indicated a requirement for $\mathrm{CD} 4+\mathrm{T}$ cells in the expansion of OT-I T cells via anti-mouse OX40 mAb. In those experiments in Major Histocompatibility Complex (MHC) Class II KO mice, a reduction in the resting memory population was observed, with no significant change at the peak of the primary response as we show here with the mIgG2a. There are many differences in experimental set up, including the number of OT-I cells transferred, and site of priming, as well as the isotype used. Experiments performed by Ruby et $a l^{43}$ used the anti-mOX40 mAb OX86 which is a Rat IgG1 (rIgG1). rIgG1 interacts with only Fc $\gamma$ RIIb and Fc $\gamma$ RIII, giving it a low activatory Fc $\gamma R$ : inhibitory Fc $\gamma \mathrm{R}$ binding (A:I) ratio, in contrast to the mIgG2a which interacts strongly with all activatory Fc $\gamma \mathrm{R}$ and has a higher A:I ratio. ${ }^{44}$ Thus, while the mIgG2a is likely to mediate its effects through depletion, based on strong activatory Fc $\gamma \mathrm{R}$ interactions, the rIgG1 through its relatively greater interaction with FcyRIIb is likely to have the additional capacity of direct agonism. Hence in the MHC Class II KO mice, there is the possibility for the OX86 rIgG1 to directly agonize the transferred OT-I T cells via FcyRIIbmediated crosslinking, unlike the ahOX40 mIgG2a when used in WT recipients, potentially explaining the disparity between the two data sets.

Enumerating the subpopulations of $\mathrm{T}$ cells within the spleen of $\mathrm{hOX} 40^{+/+} \mathrm{KI}$ mice revealed that the mIgG2a anti-hOX40 mAb uniquely caused depletion of Tregs with lower levels of depletion seen in the CD4+ effector population. In contrast, the mIgG1 anti-hOX40 mAb caused expansion of all T-cell populations, most significantly in the general CD8+ and OT-I populations. Assuming the presence of relevant effector cells and normal distribution of Fc $\gamma$ Rs in our OT-I model, it is likely that differential Fc $\gamma \mathrm{R}$ interactions of the different isotypes explains their disparate effects. Furthermore, the superior affinity for the activatory Fc $\gamma \mathrm{R}$ and resultant deletion of suppressive Treg cells seen in mice treated with mIgG2a mAb likely explain the mechanism behind the expansion of OT-I cells in the blood and spleen of hOX $40^{+/+} \mathrm{KI}$ but not WT mice. 
Another disparity seen between the anti-hOX40 mIgG1 and mIgG2a-treated mice was the recall response to SIINFEKL peptide in the OT-I model. Considering the similar levels of OT-I cells at the peak of the primary response, it was surprising to observe such a difference in the frequency in the memory phase. A positive correlation between the frequency of OT-I cells pre-recall and the frequency of OT-I cells at the peak of the memory response highlighted the possibility that it was simply the result of the amount of cells present at the time of re-challenge, with mIgG1 mAb but not mIgG2a mAb providing signals for survival/persistence. Within the primary response the MPEC population $\left(\mathrm{CD} 127^{+} \mathrm{KLRG1}^{-}\right.$) was increased at day 18 in mice treated with $\mathrm{mIgG} 1 \mathrm{mAb}$ compared with those treated with mIgG2a. Those mice also generated a higher percentage of $\mathrm{CXCR} 3{ }^{\text {hi }} \mathrm{CD} 43^{\text {lo }}$ highly proliferative cells compared with mice treated with the mIgG2a mAb. These findings, alongside those relating to the frequency of antigen-specific cells prerechallenge explain the disparity in the recall response comparing mIgG1 versus mIgG2a mAb. The exact mechanisms underpinning this dichotomy are not immediately clear but one possibility is that mIgG2a deplete Tregs alongside, to a lesser extent, CD4+ effectors, to influence priming. Importantly though, this higher proportion of effector cells in mIgG2a-treated mice versus highly proliferative cells in mIgG1-treated mice may explain these differences in memory but moreover provide a rationale for how both isotypes are able to cause equivalent efficacy in mouse tumor models, despite exhibiting opposing mechanisms of action.

Analysis of affinity, on and off rates failed to reveal a correlation with activity. However, despite the limited numbers of antibodies against each individual domain, our data suggests a correlation between domain binding and strength of both mIgG2a depletion and mIgG1 agonism. Anti-hOX40 mAb, which bound to CRD4, were more potent agonists as $\mathrm{mIgG1}$ when compared with $\mathrm{mAb}$ which bound to CRD1-3, although testing against a wider panel of antibodies would be required to strengthen this finding. This directly contrasts with our previous observations with anti-CD40 $\mathrm{mAb}^{16}$ where CRD1 binding $\mathrm{mAb}$ were more agonistic. However, for both the anti-hCD40 and anti-hOX40 mAb tested, optimal agonistic function correlated with binding outside the natural ligand binding region. This potentially suggests that the combined effects of both the ligand and the mAb in clustering the receptor are required to elicit optimal agonism. This conjecture is partially supported by recent evidence from Zhang et $a l^{17}$ who also demonstrated strong agonistic function with a CRD4-binding anti-mOX40 mAb. Those authors also documented equivalent agonistic activity with a CRD2binding, ligand-blocking anti-mOX $40 \mathrm{mAb}$ indicating the fine epitope is also important, as we reported previously for anti-CD $40 .^{16}$

The ability of TNFR targeting mAb to cause depletion of intratumoral Tregs has been clearly demonstrated in recent years, ${ }^{8} 1245$ both increasing their therapeutic possibilities but confusing potential mechanisms of action. To assess, in a more translational setting, the depleting ability of our anti-hOX $40 \mathrm{mAb}$, we performed depletion experiments in NSG mice engrafted with human target cells. In these experiments, hOX40 and other TNFR family members become upregulated on the activated Treg (online supplemental figure $5 \mathrm{~B}^{13}$ ), enabling them to serve as targets akin to those seen in tumor samples. When compared with the clinically relevant Treg depletor Yervoy, ${ }^{47}$ SAP 9 hIgG1 was not only as strong a Treg depletor but also generated a higher CD8:Treg ratio, indicating the therapeutic potential of this anti-hOX $40 \mathrm{mAb}$. Importantly, deletion of Treg was specific even though all T-cell subsets had expanded and were activated through the NSG passage.

Having established the agonistic and depletory capacity of our mAb, it was then perhaps slightly surprising for both isotypes to act therapeutically to a similar extent in tumor models but likely reflects their relative propensity to elicit different effector functions in the TME. The latter is known to have profound effects on therapeutic efficacy. ${ }^{48}$ A tumor with a high infiltrate of cells expressing activatory FcrR such as NK cells and macrophages, is likely to be more responsive to a mIgG2a mAb and depletion of detrimental target cells than a T-cell-agonizing mIgG1 mAb. Conversely, if the inhibitory Fc $\gamma$ RIIB is more prevalent, mIgG1-mediated agonism may be more prominent. Lymphoid organs outside the tumor may also be relevant. In the E.G7-OVA model, although the magnitude of the effect differed, the mIgG1 mAb largely caused T-cell expansion and the mIgG2a depletion in spleen and draining lymph nodes. It seems likely therefore that these intrinsic differences underpin the relevant mechanism of action in each case. Accordingly, mIgG2a reagents likely achieve therapeutic effects through depleting Tregs, releasing T-cell effector responses whereas mIgG1 expand T-cell numbers and hence increase cytotoxic effectors within the tumor site. Despite the disparity in recall responses in the OT-I model (mIgG1 >>mIgG2a), in the E.G7-OVA model both isotypes were able to elicit tumor control and generate memory sufficient to prevent tumor growth on rechallenge. This suggests that other factors must be operational in the presence of tumor. One possibility is that the threshold for memory is relatively low in the presence of an immunogeneic tumor and that the limited level of memory recall seen following treatment with the mIgG2a is sufficient. Alternatively, it may indicate that the mIgG2a mechanism of action, deleting Tregs, allows the expansion of otherwise cryptic epitopes allowing T-cell control as was previously described in the CT26 model. ${ }^{49}$

In recent years, evidence for cytotoxic CD4+ T cells in tumor eradication has been reported. ${ }^{50}{ }^{51}$ Through the use of both adoptive transfer models and the B16 F10 melanoma model, Qui et a $\iota^{51}$ showed that OX40 mAb stimulation increased GzmB production in CD4+ T cells and that dual co-stimulation with 4-1BB could expand these cells. In our E.G7 model, however, we failed to 
see consistent increases in either $\mathrm{CD} 4+\mathrm{GzmB}+\mathrm{T}$ cells or $\mathrm{CD} 4+$ Eomes+T-bet+ $\mathrm{T}$ cells in response to anti-OX40 $\mathrm{mAb}$ (online supplemental figure $7 \mathrm{~A}-\mathrm{C}$ ) regardless of isotype used. This potentially reflects the differences in how GzmB was measured in the two tumor models as in the B16 F10 model, isolated cells were stimulated for 24 hours with anti-CD3 mAb prior to GzmB detection. As we measured GzmB directly ex vivo, we cannot rule out that on restimulation we would also have revealed CD4+ cytotoxic potential in response to OX40 stimulation.

In summary, our findings show that immunomodulatory $\mathrm{mAb}$ directed against hOX40 can harness multiple mechanisms of action to elicit tumor control. They also show that these mechanisms can be modulated dependent on the choice of isotype and domain-binding region. Targeting the membrane proximal domains appears optimal for both deletion and agonism; with the latter strongly driven by isotypes with low A:I ratios such as mIgG1. Lowering the A:I ratio can be attained in many different ways, for example, by increasing the affinity for Fc $\gamma$ RIIB, which has been shown to mediate more effective agonism for anti-CD40 $\mathrm{mAb}^{52}$ and a recent paper showed that this may also be true for OX40 using a clinically relevant antibody ${ }^{53}$ in vitro but as yet it is unclear whether this will be true in vivo for anti-hOX40 mAb. These findings have implications for the design of the next generation of anti-hOX $40 \mathrm{mAb}$ for the clinic. Current reagents typically display an unmodified hIgG1 isotype and although safe have not delivered strong anti-tumor effects. ${ }^{6}$ These hIgG1 reagents would be expected to deliver the Tregdeleting function indicated here but to date this activity has not been shown clearly in patients. Furthermore, their deletion may not be sufficient to elicit tumor regression in most human cancers, unlike the mouse models shown here. Therefore, $\mathrm{mAb}$ with appropriate isotypes and further engineering (hIgG2B, ${ }^{16} 54$ SELF $^{52} 55$ and

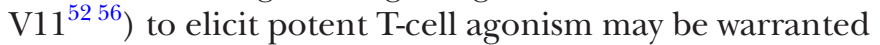
for further investigation for use both as a monotherapy but more importantly in combination.

\section{METHODS AND MATERIALS Human samples}

PBMCs were obtained from healthy adult volunteers from either Southampton National Blood Service, UK, or Hallands Hospital Halmstad, Sweden. For NSG reconstitution experiments performed in Southampton, hPBMCs were purchased from STEMCELL Technologies.

\section{Mice}

C57BL/6 mice and OT-I transgenic mice were obtained from Charles River Laboratories. NSG mice were purchased from Jackson Laboratories. hOX40 KI mice were generated by Ozgene. hOX40/OT-I mice were generated in-house. For all experiments, young adult mice were sex-matched and age-matched and randomly assigned to experimental groups. Experiments were not blinded.

\section{Antibody production and labeling}

Anti-hOX $40 \mathrm{mAb}$ were produced and labeled using standard techniques as detailed in online supplemental material 1. Cetuximab was a kind gift from Thomas Valerius and Campath-1 was a kind gift from Geoff Hale.

\section{Humanization of SAP 9}

The variable regions of SAP 9 heavy and light chains were sequenced from the hybridoma by PCR. The sequence was humanized using Macromoltek's proprietary humanisation algorithms. A generic antibody signal peptide sequence was then added to the humanized variable region sequences and the amino acid sequences converted into nucleotide sequences using https://www. bioinformatics.org/sms2/rev_trans.html. Nucleotide sequences were synthesized by GeneArt and subcloned into expression vector pEE6.4 (Lonza) for expression.

Binding domain determination/blocking experiments hOX 40 constructs were transiently transfected into $293 \mathrm{~F}$ cells before addition of $10 \mu \mathrm{g} / \mathrm{mL}$ anti-hOX $40 \mathrm{mAb}$, binding was detected with a PE-labeled secondary antimouse Fc antibody (Jackson Laboratories). For blocking experiments, unlabeled antibody was added for $30 \mathrm{~min}$ before addition of FITC-labeled anti-hOX40 mAb. Binding was assessed using flow cytometry (see below).

\section{Surface plasmon resonance}

A Biacore T100 upgraded to a T200 (GE Life Sciences) was used to measure interactions with hOX40. $100 \mathrm{nM}$ of hOX40L-His was immobilized onto a CM5 chip (GE Healthcare) coated with an anti-His mAb. $100 \mathrm{nM}$ hOX 40-hFc was subsequently captured followed by the injection of anti-hOX40 mAb $(15 \mu \mathrm{g} / \mathrm{mL})$.

\section{In vitro assays}

Standard hPBMC proliferation assays were performed as detailed in online supplemental material 1. For expression assays, frozen hPBMCs were thawed and rested overnight, then stimulated with plate-bound anti-CD3 (OKT3, $15 \mathrm{ng} / \mathrm{mL})$ and soluble anti-CD28 (CD28.2, $0.5 \mu \mathrm{g} / \mathrm{mL})$. Cells were harvested, stained with appropriate antibodies and assessed via flow cytometry.

Murine expression assays; standard activation was used as detailed in online supplemental material 1. Cells were stained with appropriate antibodies and analyzed by flow cytometry.

\section{Flow cytometry}

Standard staining and analysis performed as detailed in online supplemental material 1. Antibodies used are detailed in online supplemental table 1.

\section{OT-I adoptive transfer}

A total of $1 \times 10^{5} \mathrm{hOX} 40 \mathrm{KI}^{+/-}$OT-I cells were injected intravenously into hOX $40 \mathrm{KI}^{+/+}$or WT C57BL/ 6 mice. Twentyfour hours later $5 \mathrm{mg}$ ovalbumin (Sigma) and $100 \mu \mathrm{g}$ anti-hOX40 or isotype control were given intraperitoneally (i.p.). Deletion was determined by harvesting spleens day 4 post i.p. injection. OT-I kinetics were monitored in 
the blood through SIINFEKL tetramer staining and mice were rechallenged between 6 and 10 weeks later with $30 \mathrm{nM}$ SIINFEKL intravenously. Based on preliminary experiments, $n=3$ was determined as sufficient to see a $\mathrm{p}<0.05$ for OT-I expansion. Mice with SIINFEKL tetramer responses less than $1 \%$ of $\mathrm{CD} 8+$ lymphocytes at the peak of the response were excluded due to the likelihood that the OT-I transfer had failed since isotype controls peak at an average $5.2 \% \pm 0.58 \mathrm{SEM}$ (mIgG1) and $4.65 \% \pm 0.65$ SEM (mIgG2a) in blood and $3.8 \% \pm 0.79$ SEM (mIgG1) and $3.1 \% \pm 0.89$ on Day 4 in spleens. One mouse was excluded from figure 4B groups SAP-28, 15-3 and 9 based on this criteria.

\section{Treg cell depletion in reconstituted NOG/SCID mice}

PBMC-NOG/severe combined immune-deficient (SCID) mice (primary human xenograft model) were generated by injecting NOG or NSG mice with $1-1.5 \times 10^{7}$ PBMC isolated using Ficoll-Paque PLUS. Approximately 2 weeks after reconstitution of NOG or NSG mice with hPBMCs, spleens were harvested. Splenocytes $10 \times 10^{6}$ were then injected into the peritoneal cavity of naive SCID or NSG mice 1 hour prior to injection with $10 \mathrm{mg} / \mathrm{kg}$ of depleting $\mathrm{mAb}$ or isotype control mAb. The peritoneal fluid was collected after 24 hours and human T-cell subsets were identified by flow cytometry.

\section{Tumor models}

E.G7-OVA and MCA-205 tumor models: $5 \times 10^{5}$ tumor cells were injected subcutaneously into the flank of mice. Based on preliminary experiments, $n=5$ was determined as sufficient to see a $\mathrm{p}<0.05$ for tumor therapy. Groups of eight (eg, 7-OVA) or six (MCA-205) mice were set up to ensure that at treatment there would be a minimum of 5 per group with established tumors with comparable size (between $5 \times 5 \mathrm{~mm}$ and $8 \times 8 \mathrm{~mm}$ ). Mice were then ranked according to tumor size and assigned to treatments groups so that average tumor size per group was similar prior to treatment. This ensured mixed treatment groups within cages to reduce influence of housing on treatment effect. Established tumors were treated with $3 \times 100 \mu \mathrm{g}$ anti-hOX $40 \mathrm{mAb}$ or isotype i.p. every other day. For phenotyping experiments, organs were harvested day 4 post final injection. Tumors were digested using 0.5 units of liberase TL (Roche) and cells analyzed via flow cytometry. For survival experiments, tumor size mice were culled once they reached a terminal size $(\mathrm{eg}, 7: 20 \times 20 \mathrm{~mm}$, MCA-205: $15 \times 15 \mathrm{~mm})$. Mice which eradicated tumor after treatment were rechallenged with $5 \times 10^{5}$ tumor cells subcutaneously into the flank.

\section{Statistics}

All results show mean \pm SEM. One-way Anova with multiple comparisons (Dunnett's, Tukey's or Sidak's as stated in legend) or Mann-Whitney tests were used as stated in legends performed using GraphPad Prism. Survival curves evaluated using a Log-rank (Mantel-Cox) test. Significance is shown relative to isotype control unless bar is shown. Where indicated, ns $=$ not significant, ${ }^{*} \mathrm{p} \leq 0.05$, $* * \mathrm{p} \leq 0.01, * * * \mathrm{p} \leq 0.001, * * * * \mathrm{p} \leq 0.0001$.

Acknowledgements We are grateful to the staff of the University of Southampton Biomedical Research facility for their technical support. We also thank Leon Douglas and Patrick Duriez from the Experimental Cancer Medicine Centre/Cancer Research UK-funded Protein Core Facility for making SIINFEKL tetramers.

Contributors JG, KH, HLS, KC, RRF, HTC, TS, MS, LM and JW performed experiments. JG, KH, HLS, TS, RRF, MS, LM and JW performed statistical analyses. JG, JW, IT, BF and MSC designed experiments. JG, JW and MSC wrote the manuscript. All authors contributed to manuscript revision and read and approved the submitted version.

Funding This work was supported by Cancer Research UK (CRUK) programme grants awarded to MJG and MSC (Award number: A20537, A24721), CRUK centre grant (Award number: A25139) and a CRUK studentship to JG and MSC from the Southampton CRUK centre (Award number: A29286).

Competing interests MSC is a retained consultant for Biolnvent International and has performed educational and advisory roles for Baxalta and Boehringer Ingleheim. He has received research funding from Roche, Gilead, Bioinvent International and GSK. BF, IT, LM and MS are employees of Bioinvent International.

Patient consent for publication Not required.

Ethics approval All procedures were conducted in accordance with UK Home Office guidelines and were approved by the University of Southampton's ethical committee or at Biolnvent under Dnr 14760/2016. For clinical samples, ethical approval was obtained from the Ethics Committee of Skåne University Hospital, Sweden.

Provenance and peer review Not commissioned; externally peer-reviewed.

Data availability statement Data are available upon reasonable request. All data sets used and/or analyzed during the current study are available from the corresponding author on reasonable request.

Supplemental material This content has been supplied by the author(s). It has not been vetted by BMJ Publishing Group Limited (BMJ) and may not have been peer-reviewed. Any opinions or recommendations discussed are solely those of the author(s) and are not endorsed by BMJ. BMJ disclaims all liability and responsibility arising from any reliance placed on the content. Where the content includes any translated material, BMJ does not warrant the accuracy and reliability of the translations (including but not limited to local regulations, clinical guidelines, terminology, drug names and drug dosages), and is not responsible for any error and/or omissions arising from translation and adaptation or otherwise.

Open access This is an open access article distributed in accordance with the Creative Commons Attribution 4.0 Unported (CC BY 4.0) license, which permits others to copy, redistribute, remix, transform and build upon this work for any purpose, provided the original work is properly cited, a link to the licence is given, and indication of whether changes were made. See https://creativecommons.org/ licenses/by/4.0/.

ORCID iDs

Jane E Willoughby http://orcid.org/0000-0002-6326-4519

Mark S Cragg http://orcid.org/0000-0003-2077-089X

\section{REFERENCES}

1 Seidel JA, Otsuka A, Kabashima K. Anti-Pd-1 and anti-CTLA-4 therapies in cancer: mechanisms of action, efficacy, and limitations. Front Oncol 2018;8:86.

2 Dine J, Gordon R, Shames Y, et al. Immune checkpoint inhibitors: an innovation in immunotherapy for the treatment and management of patients with cancer. Asia Pac J Oncol Nurs 2017;4:127-35.

3 Remer M, White A, Glennie M, et al. The use of anti-CD40 mAb in cancer. Curr Top Microbiol Immunol 2017;405:165-207.

4 Bartkowiak T, Curran MA. 4-1Bb agonists: multi-potent potentiators of tumor immunity. Front Oncol 2015;5:117.

5 Aspeslagh S, Postel-Vinay S, Rusakiewicz S, et al. Rationale for antiOX40 cancer immunotherapy. Eur J Cancer 2016;52:50-66.

6 Buchan SL, Rogel A, Al-Shamkhani A. The immunobiology of CD27 and OX40 and their potential as targets for cancer immunotherapy. Blood 2018;131:39-48.

7 Kuang Z, Jing H, Wu Z, et al. Development and characterization of a novel anti-OX40 antibody for potent immune activation. Cancer Immunol Immunother 2020;69:939-50. 
8 Freeman ZT, Nirschl TR, Hovelson DH, et al. A conserved intratumoral regulatory $T$ cell signature identifies $4-1 \mathrm{BB}$ as a pancancer target. J Clin Invest 2020;130:1405-16.

9 Croft M. Costimulation of T cells by OX40, 4-1BB, and CD27. Cytokine Growth Factor Rev 2003;14:265-73.

10 Li F, Ravetch JV. A general requirement for FcyRIIB co-engagement of agonistic anti-TNFR antibodies. Cell Cycle 2012;11:3343-4.

11 White ALet al. FcgammaRlotalotaB controls the potency of agonistic anti-TNFR mAbs. Cancer Immunol Immunother 2013;62:941-8.

12 Bulliard Y, Jolicoeur R, Zhang J, et al. Ox40 engagement depletes intratumoral Tregs via activating FcyRs, leading to antitumor efficacy. Immunol Cell Biol 2014;92:475-80.

13 Buchan SL, Dou L, Remer M, et al. Antibodies to costimulatory receptor 4-1BB enhance anti-tumor immunity via T regulatory cell depletion and promotion of CD8 T cell effector function. Immunity 2018;49:958-70.

14 Weinberg AD, Morris NP, Kovacsovics-Bankowski M, et al. Science gone translational: the OX40 agonist story. Immunol Rev 2011;244:218-31.

15 Cleary KLS, Chan HTC, James S, et al. Antibody distance from the cell membrane regulates antibody effector mechanisms. J Immunol 2017;198:3999-4011.

16 Yu X, Chan HTC, Orr CM, et al. Complex interplay between epitope specificity and isotype dictates the biological activity of anti-human CD40 antibodies. Cancer Cell 2018;33:664-75.

17 Zhang P, Tu GH, Wei J, et al. Ligand-Blocking and membraneproximal domain targeting Anti-OX40 antibodies mediate potent $\mathrm{T}$ Cell-Stimulatory and anti-tumor activity. Cell Rep 2019;27:3117-23.

18 Bulliard Y, Jolicoeur R, Windman M, et al. Activating Fc $\gamma$ receptors contribute to the antitumor activities of immunoregulatory receptortargeting antibodies. J Exp Med 2013;210:1685-93.

19 Fujita T, Ukyo N, Hori T, et al. Functional characterization of OX40 expressed on human CD8+ T cells. Immunol Lett 2006;106:27-33.

20 Xie F, Wang Q, Chen Y, et al. Characterization and application of two novel monoclonal antibodies against human OX40: costimulation of $T$ cells and expression on tumor as well as normal gland tissues. Tissue Antigens 2006;67:307-17.

21 Compaan DM, Hymowitz SG. The crystal structure of the costimulatory OX40-OX40L complex. Structure 2006;14:1321-30.

22 Willoughby J, Griffiths J, Tews I, et al. OX40: structure and function What questions remain? Mol Immunol 2017;83:13-22.

23 Croft M. Control of immunity by the TNFR-related molecule OX40 (CD134). Annu Rev Immunol 2010;28:57-78.

24 Beers SA, Glennie MJ, White AL. Influence of immunoglobulin isotype on therapeutic antibody function. Blood 2016;127:1097-101.

25 Croft M, Benedict CA, Ware CF. Clinical targeting of the TNF and TNFR superfamilies. Nat Rev Drug Discov 2013;12:147-68.

26 Joshi NS, Cui W, Chandele A, et al. Inflammation directs memory precursor and short-lived effector CD8(+) T cell fates via the graded expression of T-bet transcription factor. Immunity 2007;27:281-95.

27 Bjorkdahl O, Barber KA, Brett SJ, et al. Characterization of CCchemokine receptor 7 expression on murine T cells in lymphoid tissues. Immunology 2003;110:170-9.

28 Kapasi ZF, Murali-Krishna K, McRae ML, et al. Defective generation but normal maintenance of memory T cells in old mice. Eur $J$ Immunol 2002;32:1567-73.

29 Hikono H, Kohlmeier JE, Takamura S, et al. Activation phenotype, rather than central- or effector-memory phenotype, predicts the recall efficacy of memory CD8+ T cells. J Exp Med 2007;204:1625-36.

30 Olson JA, McDonald-Hyman C, Jameson SC, et al. Effector-like CD8 T cells in the memory population mediate potent protective immunity. Immunity 2013;38:1250-60.

31 Ataera H, Simkins HMA, Hyde E, et al. The control of CD8+ T cell responses is preserved in perforin-deficient mice and released by depletion of CD4+CD25+ regulatory T cells. J Leukoc Biol 2013;94:825-33.

32 Heit A, Gebhardt F, Lahl K, et al. Circumvention of regulatory CD4(+) $T$ cell activity during cross-priming strongly enhances $T$ cellmediated immunity. Eur J Immunol 2008;38:1585-97.

33 McNally A, Hill GR, Sparwasser T, et al. Cd4+Cd25+ regulatory T cells control CD8+ T-cell effector differentiation by modulating IL-2 homeostasis. Proc Natl Acad Sci U S A 2011;108:7529-34.
34 Issa F, Milward K, Goto R, et al. Transiently activated human regulatory T cells upregulate $\mathrm{BCL}-\mathrm{XL}$ expression and acquire a functional advantage in vivo. Front Immunol 2019;10:889

35 Sato E, Olson SH, Ahn J, et al. Intraepithelial CD8+ tumor-infiltrating lymphocytes and a high CD8+/regulatory $T$ cell ratio are associated with favorable prognosis in ovarian cancer. Proc Natl Acad Sci U S A 2005;102:18538-43.

36 Shimizu J, Yamazaki S, Sakaguchi S. Induction of tumor immunity by removing CD25+CD4+ T cells: a common basis between tumor immunity and autoimmunity. J Immunol 1999;163:5211-8.

37 Onizuka S, Tawara I, Shimizu J, et al. Tumor rejection by in vivo administration of anti-CD25 (interleukin-2 receptor alpha) monoclonal antibody. Cancer Res 1999;59:3128-33.

38 Intlekofer AM, Takemoto N, Wherry EJ, et al. Effector and memory CD8+ T cell fate coupled by T-bet and eomesodermin. Nat Immunol 2005;6:1236-44.

39 Pearce EL, Mullen AC, Martins GA, et al. Control of effector CD8+ $T$ cell function by the transcription factor eomesodermin. Science 2003;302:1041-3.

40 Banerjee A, Gordon SM, Intlekofer AM, et al. Cutting edge: the transcription factor eomesodermin enables CD8+ T cells to compete for the memory cell niche. J Immunol 2010;185:4988-92.

41 Bruhns P. Properties of mouse and human IgG receptors and their contribution to disease models. Blood 2012;119:5640-9.

42 Stewart R, Hammond SA, Oberst M, et al. The role of Fc gamma receptors in the activity of immunomodulatory antibodies for cancer. $J$ Immunother Cancer 2014;2:29.

43 Ruby CE, Redmond WL, Haley D, et al. Anti-OX40 stimulation in vivo enhances CD8+ memory T cell survival and significantly increases recall responses. Eur J Immunol 2007;37:157-66.

44 Arce Vargas F, Furness AJS, Solomon I, et al. Fc-Optimized AntiCD25 depletes tumor-infiltrating regulatory $T$ cells and synergizes with PD-1 blockade to eradicate established tumors. Immunity 2017:46:577-86

45 Marabelle A, Kohrt H, Sagiv-Barfi I, et al. Depleting tumor-specific Tregs at a single site eradicates disseminated tumors. J Clin Invest 2013;123:2447-63.

46 Simpson TR, Li F, Montalvo-Ortiz W, et al. Fc-Dependent depletion of tumor-infiltrating regulatory $T$ cells co-defines the efficacy of antiCTLA-4 therapy against melanoma. J Exp Med 2013;210:1695-710.

47 Arce Vargas F, Furness AJS, Litchfield K, et al. Fc effector function contributes to the activity of human anti-CTLA-4 antibodies. Cancer Cell 2018;33:649-63.

$48 \mathrm{Klemm}$ F, Joyce JA. Microenvironmental regulation of therapeutic response in cancer. Trends Cell Biol 2015;25:198-213.

49 James E, Yeh A, King C, et al. Differential suppression of tumor-specific CD8+ T cells by regulatory T cells. J Immunol 2010;185:5048-55.

50 Quezada SA, Simpson TR, Peggs KS, et al. Tumor-reactive CD4(+) T cells develop cytotoxic activity and eradicate large established melanoma after transfer into lymphopenic hosts. J Exp Med 2010;207:637-50.

51 Qui HZ, Hagymasi AT, Bandyopadhyay S, et al. Cd134 plus CD137 dual costimulation induces eomesodermin in CD4 T cells to program cytotoxic Th1 differentiation. J Immunol 2011;187:3555-64.

52 Dahan R, Barnhart BC, Li F, et al. Therapeutic activity of agonistic, human anti-CD40 monoclonal antibodies requires selective $\mathrm{Fc} \gamma \mathrm{R}$ engagement. Cancer Cell 2016;29:820-31.

53 Campos Carrascosa L, van Beek AA, de Ruiter V, et al. FcүRIIIB engagement drives agonistic activity of Fc-engineered $\alpha \mathrm{OX} 40$ antibody to stimulate human tumor-infiltrating T cells. J Immunother Cancer 2020;8:e000816.

54 White AL, Chan HTC, French RR, et al. Conformation of the human immunoglobulin G2 hinge imparts superagonistic properties to immunostimulatory anticancer antibodies. Cancer Cell 2015;27:138-48

55 Chu SY, Vostiar I, Karki S, et al. Inhibition of B cell receptormediated activation of primary human $B$ cells by coengagement of CD19 and FcyRllb with Fc-engineered antibodies. Mol Immunol 2008;45:3926-33.

56 Mimoto F, Katada H, Kadono S, et al. Engineered antibody Fc variant

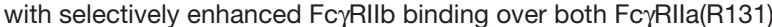
and FcyRlla(H131). Protein Eng Des Sel 2013;26:589-98. 